\title{
Data reduction methods for ektacytometry in clinical hemorheology
}

\author{
Oguz K. Baskurt ${ }^{\mathrm{a}, *}$ and Herbert J. Meiselman ${ }^{\mathrm{b}}$ \\ ${ }^{a}$ Koc University School of Medicine, Istanbul, Turkey \\ ${ }^{\mathrm{b}}$ Keck School of Medicine, University of Southern California, Los Angeles, CA, USA
}

\begin{abstract}
Laser-diffraction ektacytometry is a generally accepted technique for measuring RBC deformability induced by fluid shear stress (SS) and yields paired elongation index-SS data at several levels of stress. Unfortunately, comparison of results is hindered by the lack of simple indices that accurately characterize these data. Several mathematical models have been proposed, including those developed for analysis of enzyme kinetics (Lineweaver-Burk, Eadie-Hofstee) and curve fitting (StreekstraBronkhorst). All of these analytical approaches provide a value for cell deformation at infinite stress $\left(\mathrm{EI}_{\max }\right)$ and the shear stress required to achieve one-half of this deformation $\left(\mathrm{SS}_{1 / 2}\right)$; the use of non-linear regression is essential when calculating these parameters. While the current models provide equivalent results for normal RBC if used with non-linear regression, $\mathrm{EI}_{\max }$ and $\mathrm{SS}_{1 / 2}$ are not always concordant for cells with abnormal mechanical behavior. This technical note examines such differences for three conditions: glutaraldehyde treatment, mechanical stress and non-isotonic media. It was found that none of the models yield completely satisfactory values for $\mathrm{EI}_{\max }$ and $\mathrm{SS}_{1 / 2}$, especially if there are large changes of $\mathrm{EI}_{\max }$. However, the ratio of $\mathrm{SS}_{1 / 2}$ to $\mathrm{EI}_{\max }\left(\mathrm{SS}_{1 / 2} / \mathrm{EI}_{\max }\right)$ is much less affected by these problems, has similar power (i.e., standardized difference) as $\mathrm{SS}_{1 / 2}$ and $\mathrm{EI}_{\max }$ and is more robust in reflecting alterations of deformability. We thus conclude that the $\mathrm{SS}_{1 / 2} / \mathrm{EI}_{\max }$ ratio can be used when reporting and comparing various populations of RBC or cells obtained from subjects having different clinical states.
\end{abstract}

Keywords: Red blood cell deformability, hemorheology, data reduction, ektacytometry

\section{Introduction}

Ektacytometry is a widely used method to assess red blood cell (RBC) deformability. The usual application of the method involves the measurement of RBC elongation indexes (EI) over a range of shear stresses (SS) via shape analysis of laser diffraction images [8, 10, 17, 18]. A typical EI-SS curve is composed of 7-10 EI-SS data pairs ranging from very low (e.g., $0.5 \mathrm{~Pa}$ ) to moderately high (e.g., $50 \mathrm{~Pa}$ ) SS [1]. The relationship between EI and SS in this range is characterized by a sigmoidal curve when SS is plotted using a logarithmic scale. While this approach provides information on RBC deformability in the low and high SS ranges, it is limited by difficulties when attempting comparisons between groups. That is, one is not sure at which SS to compare and whether to focus on low, medium or high SS.

\footnotetext{
${ }^{*}$ Corresponding author: Oguz K. Baskurt, MD, PhD, Professor of Physiology, Koc University School of Medicine, Rumelifeneri Yolu, 34450, Sariyer Istanbul, Turkey. Tel.: +90 212338 1160; Fax: +90212338 1168; E-mail: obaskurt@ku.edu.tr.
} 


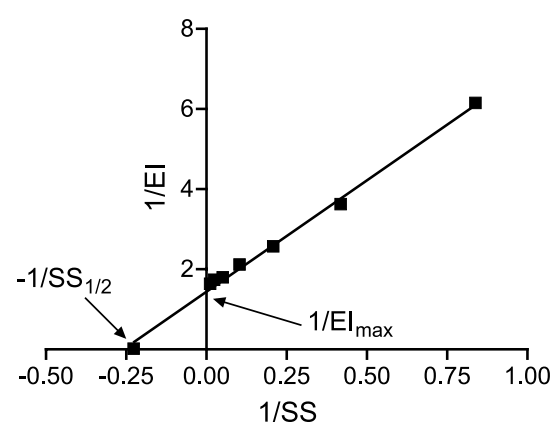

a. Lineweaver-Burk

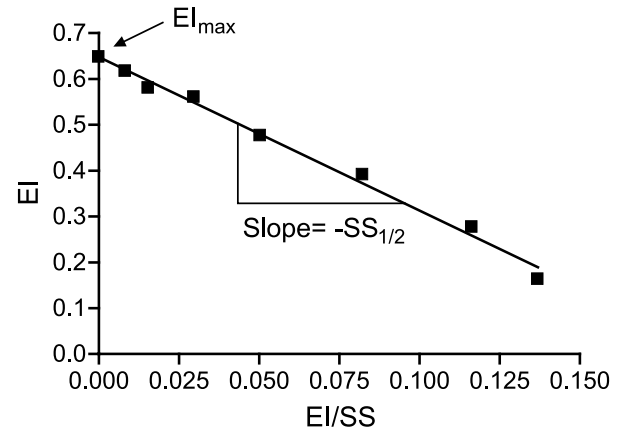

b. Eadie-Hofstee

Fig. 1. Graphical representations of Lineweaver-Burk (a) and Eadie-Hofstee (b) approaches. The same shear stress - elongation index data were used for each approach.

A single index reflecting RBC deformability would be useful, in experimental and clinical studies $[7,12$, $14,15,22]$. Various approaches have been suggested to derive indices of RBC deformability based on EI-SS curves [4, 6, 7, 19], some being implemented into software programs that are part of commercial instruments (e.g., LORCA). These approaches were critically analyzed by various groups and recommendations for their use have been published $[2,3,11]$. This technical report summarizes and discusses various problems associated with the application of these data reduction methods for ektacytometry results. Note that all data reported herein were obtained by the authors using a laser diffraction system (LORCA MaxSis, Mechatronics, Amsterdam, The Netherlands) at $37^{\circ} \mathrm{C}$ for $\mathrm{RBC}$ suspended in viscous polyvinylpyrrolidone (PVP) media (Mechatronics); unless otherwise stated all media were isotonic at $295 \mathrm{mOsm} / \mathrm{kg}$.

\section{Summary of approaches proposed for data reduction in ektacytometry}

The several approaches to the reduction of SS-EI data are based on the similarity of the shape of SSEI curves to Michaelis-Menten type enzyme kinetics curves, and similar methods have been applied in analyzing both types of curves [6, 19]. These include the Lineweaver-Burk [13] and the Eadie-Hofstee approaches [9]: both assume that EI reaches a theoretical maximum $\left(\mathrm{EI}_{\max }\right)$ at infinite $\mathrm{SS}$, and that a SS value required to achieve one-half of $\mathrm{EI}_{\max }$ similar to a $\mathrm{Km}$ value in enzyme kinetics, can be calculated.

In the Lineweaver-Burk (LB) approach, the reciprocals of EI and SS data are plotted and the ordinate intercept gives $1 / \mathrm{EI}_{\max }$ and the abscissa intercept corresponds to the negative of $1 / \mathrm{SS}_{1 / 2}$ (Fig. 1a). This linear relationship is represented by Equation 1; note that the term $\left(\mathrm{SS}_{1 / 2} / \mathrm{EI}_{\max }\right)$ is the slope of the line:

$$
\frac{1}{E I}=\frac{S S_{1 / 2}}{E I_{\max }} \times \frac{1}{S S}+\frac{1}{E I_{\max }}
$$

In Eadie-Hofstee (EH) approach, the EI data are plotted against EI/SS, again yielding a linear relationship, with the ordinate intercept corresponding to $\mathrm{EI}_{\max }$ and the slope of the line the negative value of $\mathrm{SS}_{1 / 2}$ (Fig. 1b). The relationship is expressed by Equation 2:

$$
E I=E I_{\max }-\frac{E I}{S S} \times S S_{1 / 2}
$$


It can easily be demonstrated that both Equations 1 and 2 can be solved to yield Equation 3 and are therefore mathematically equivalent to each other:

$$
E I=\frac{E I_{\max } \cdot S S}{S S_{1 / 2}+S S}
$$

Despite the mathematical similarity between the LB and EH approaches, the results obtained by these methods may differ if they are applied graphically (i.e., linear regression). That is, due to the inverse forms of EI and SS in the LB approach, errors at points corresponding to the low SS (at which the variation in EI is higher compared to higher SS) are exaggerated, thereby yielding significant deviations of the fitted line [2,11]; the EH method is more robust inasmuch as it gives equal weight to data points over the entire SS range. However, the LB and $\mathrm{EH}$ approaches yield exactly the same results if the $\mathrm{EI}_{\max }$ and $\mathrm{SS}_{1 / 2}$ parameters are calculated for the same SS-EI data set, by curve-fitting to Equations 1 and 2 using non-linear curve fitting algorithms.

A third approach to data analysis is the Streekstra-Bronkhorst (SB) equation, an empirical equation fitted to the SS-EI curve. The original approach by Streekstra et al. was based on curve fitting to yield a sigmoidal curve [20]:

$$
E I=\frac{E I_{\max }}{\left(\frac{S S_{1 / 2}}{S S}\right)^{m}+1}
$$

where $\mathrm{m}$ is an exponent that indicates the steepness of the curve. It should be noted that rearrangement of the LB equation (Equation 1) yields a very similar expression:

$$
E I=\frac{E I_{\max }}{\frac{S S_{1 / 2}}{S S}+1}
$$

The only difference between Equations 4 and 5 is the presence or absence of the exponent " $m$ ". Therefore, the LB equation is a special form of the Streekstra equation (Equation 4) in which $\mathrm{m}=1$.

The Streekstra model was modified by Bronkhorst et al. [4] to yield the Streekstra-Bronkhorst (SB) equation in which an $\mathrm{EI}_{\min }$ term was added that allows $\mathrm{EI}$ to have negative values at low $\mathrm{SS}$ :

$$
E I=\left(E I_{\max }-E I_{\min }\right) \frac{\left(\frac{S S}{S S_{1 / 2}}\right)^{m}}{\left(\frac{S S}{S S_{1 / 2}}\right)^{m}+1}+E I_{\min }
$$

The $\mathrm{SS}_{1 / 2}$ in Equation 6 corresponds to the $\mathrm{SS}$ where $\mathrm{EI}$ is halfway between $\mathrm{EI}_{\max }$ and $\mathrm{EI}_{\min }$, exactly as described by the $\mathrm{LB}$ equation if $\mathrm{EI}_{\min }=0$. Hence, the model known as the $\mathrm{SB}$ equation (Equation 6) contains four parameters to describe the SS-EI curves: $\mathrm{EI}_{\max }, \mathrm{EI}_{\min }, \mathrm{SS}_{1 / 2}$ and $\mathrm{m}$. All of these four factors are affected by the shape of the SS-EI curve. Note that Equation 6 can be simplified by setting $\mathrm{EI}_{\min }$ to be zero (i.e., the curve starts at the origin) and $m$ equal to one; under these conditions, Equation 6 is then mathematically equivalent to the Lineweaver-Burk equation (Equations 1 and 5).

\section{Discrepancies between various approaches}

The application of the above-mentioned approaches to a given SS-EI data set may sometimes yield significant differences for the calculated parameters. One example arises for RBC with reduced deformability achieved by treatment with low concentrations of glutaraldehyde (GA). RBC from the same group 


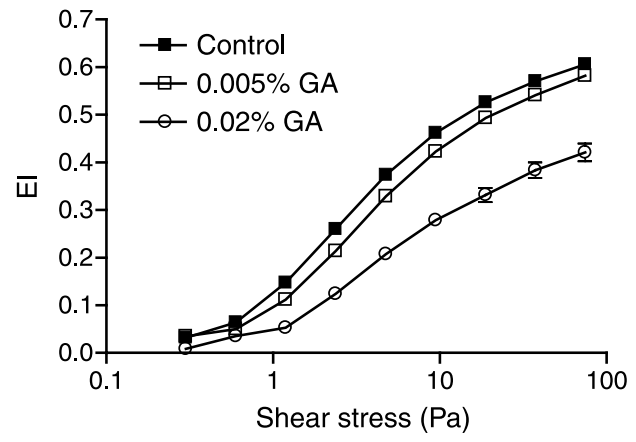

Fig. 2. Shear stress - elongation index (EI) curves for human red blood cells treated with glutaraldehyde (GA) at $0.005 \%$ and $0.02 \%$ concentrations compared with untreated (Control) RBC. Data are presented as mean \pm standard error; $n=10$. Error bars for most points remain in the symbols.

Table 1

Parameters describing shear stress-elongation index relationships for glutaraldehyde (GA) treated RBC calculated using Lineweaver-Burk and Streekstra-Bronkhorst models*

\begin{tabular}{lccccccr}
\hline & \multicolumn{3}{c}{ Lineweaver-Burk } & & \multicolumn{3}{c}{ Streekstra-Bronkhorst } \\
\cline { 2 - 3 } \cline { 6 - 7 } & Control & $0.005 \% \mathrm{GA}$ & $0.02 \% \mathrm{GA}$ & & Control & $0.005 \% \mathrm{GA}$ & $0.02 \% \mathrm{GA}$ \\
\hline $\mathrm{EI}_{\max }$ & 0.633 & 0.616 & 0.453 & & 0.623 & 0.598 & 0.449 \\
$\mathrm{SS}_{1 / 2}$ & 3.658 & 4.595 & 6.378 & & 3.017 & 4.047 & 5.478 \\
$\mathrm{EI}_{\min }$ & & & & -0.038 & -0.012 & -0.020 \\
$\mathrm{~m}$ & & & & 1.003 & 1.076 & 0.970 \\
\hline
\end{tabular}

*Parameters are mean values calculated using the data presented in Fig. 2 by a non-linear curve fitting algorithm (Prism 4.0).

of donors were incubated at room temperature with $0.005 \%$ and $0.02 \% \mathrm{GA}$ at a $0.05 \mathrm{~L} / \mathrm{L}$ hematocrit for 30 minutes, and then tested by ektacytometry; EI-SS results are shown in Fig. 2. Table 1 presents curve fitting parameters calculated using these data sets for the normal and GA-treated RBC shown in Fig. 2. Curve fitting to LB and SB equations were done by a non-linear curve fitting algorithm (Prism 4.0, GraphPad Software Inc., La Jolla, CA). Obviously, the $\mathrm{SS}_{1 / 2}$ parameters provided by the $\mathrm{LB}$ and $\mathrm{SB}$ approaches are not identical; this inequality results from the contributions of the other two factors (i.e., $\mathrm{EI}_{\min }$ and $\mathrm{m}$ ) in the SB equation.

It is notable that the differences in Table 1 between the same parameters (i.e., $\mathrm{EI}_{\max }, \mathrm{SS}_{1 / 2}$ ) calculated using different approaches may be exaggerated if the shapes of the SS-EI curves strongly diverge from the "normal" sigmoidal curve. For example, Fig. 3 presents SS-EI curves for human RBC before and after exposure to $100 \mathrm{~Pa}$ shear stress for 300 seconds. The applied mechanical stress resulted in decreased deformability as reflected by lower EI values in the range of 2-75 Pa. Interestingly, the $\mathrm{EI}_{\max }$ and $\mathrm{SS}_{1 / 2}$ parameters calculated using the LB and SB models were affected differently: $\mathrm{EI}_{\max }$ values calculated using the LB approach decreased significantly while $\mathrm{SS}_{1 / 2}$ changed only slightly (Table 2). In contrast, the $\mathrm{SB}$ approach yielded $\mathrm{SS}_{1 / 2}$ values that were three-fold greater than control while $\mathrm{EI}_{\max }$ remained relatively unaltered (Table 2 ).

Another example is presented in Fig. 4 and Table 3 for normal human RBC suspended in the viscous PVP media with different levels of osmolality: isotonic $(295 \mathrm{mOsm} / \mathrm{kg})$; hypotonic $(164 \mathrm{mOsm} / \mathrm{kg})$; 


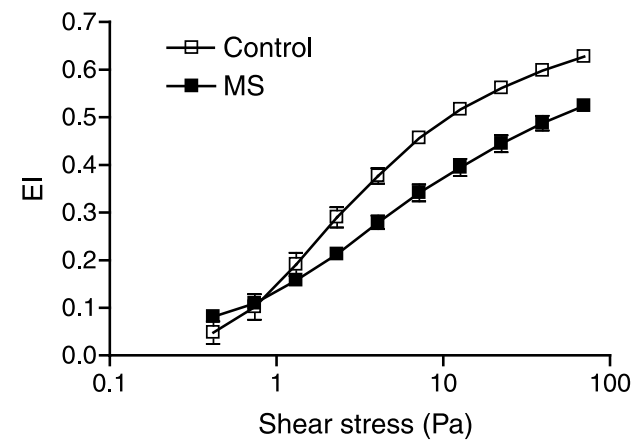

Fig. 3. Shear stress - elongation index (EI) curves for RBC before and after exposure to mechanical stress (MS) of $100 \mathrm{~Pa}$ for 300 seconds. Mean \pm SE for 14 experiments.

Table 2

Parameters describing shear stress-elongation index relationships for RBC before and after application of mechanical stress (100 Pa SS for 300 seconds) as calculated using the Lineweaver-Burk and Streekstra-Bronkhorst models*

\begin{tabular}{lccrr}
\hline & \multicolumn{2}{c}{ Lineweaver-Burk } & \multicolumn{2}{c}{ Streekstra-Bronkhorst } \\
\cline { 2 - 3 } & Control & Mechanical stress & Control & Mechanical stress \\
\hline $\mathrm{EI}_{\max }$ & 0.651 & 0.526 & 0.663 & 0.633 \\
$\mathrm{SS}_{1 / 2}$ & 3.430 & 3.769 & 2.299 & 6.836 \\
$\mathrm{EI}_{\min }$ & & & -0.121 & -0.020 \\
$\mathrm{~m}$ & & & 0.842 & 0.734 \\
\hline
\end{tabular}

*Parameters are mean values calculated using the data presented in Fig. 3 by a non-linear curve fitting algorithm (Prism 4.0).

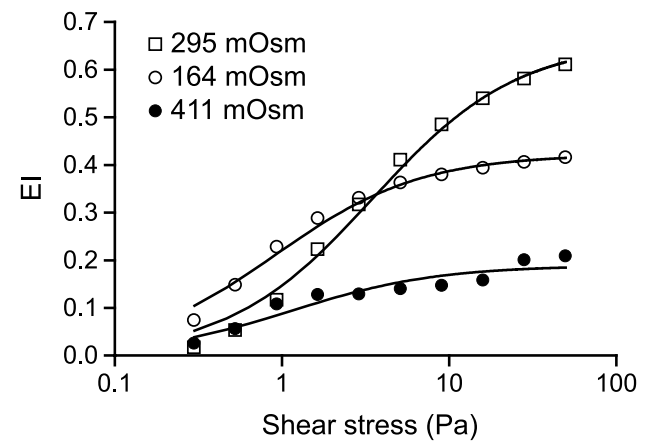

Fig. 4. Shear stress - elongation index (EI) curves measured at three osmolalities $(164 \mathrm{mOsm} / \mathrm{kg}, 295 \mathrm{mOsm} / \mathrm{kg}$ and $411 \mathrm{mOsm} / \mathrm{kg}$ ). The solid lines represent fitted curves obtained by the Lineweaver-Burk approach.

hypertonic $(411 \mathrm{mOsm} / \mathrm{kg})$. It is obvious that the non-isotonic curves markedly deviate from "normal" (i.e., control, $295 \mathrm{mOsm} / \mathrm{kg}$ ) with $\mathrm{EI}_{\max }$ decreased in both. Curve fitting parameters using the LB and SB equations confirmed the decreases in $\mathrm{EI}_{\max }$ with the calculated $\mathrm{EI}_{\max }$ values in reasonable agreement. Conversely, the $\mathrm{SS}_{1 / 2}$ parameters calculated using the two equations differ markedly (Table 3). 
Table 3

Parameters describing shear stress-elongation index relationships for RBC at three osmolalities calculated using Lineweaver-Burk and Streekstra-Bronkhorst models*

\begin{tabular}{lccccccc}
\hline & \multicolumn{3}{c}{ Lineweaver-Burk } & & \multicolumn{3}{c}{ Streekstra-Bronkhorst } \\
\cline { 2 - 3 } & $295 \mathrm{mOsm} / \mathrm{kg}$ & $164 \mathrm{mOsm} / \mathrm{kg}$ & $411 \mathrm{mOsm} / \mathrm{kg}$ & & $295 \mathrm{mOsm} / \mathrm{kg}$ & $164 \mathrm{mOsm} / \mathrm{kg}$ & $411 \mathrm{mOsm} / \mathrm{kg}$ \\
\hline $\mathrm{EI}_{\max }$ & 0.659 & 0.422 & 0.189 & & 0.637 & 0.420 & 0.253 \\
$\mathrm{SS}_{1 / 2}$ & 3.494 & 0.913 & 1.152 & & 2.507 & 0.333 & 0.003 \\
$\mathrm{EI}_{\min }$ & & & & -0.064 & -0.247 & -1.473 \\
$\mathrm{~m}$ & & & & & 1.008 & 0.856 & 0.287 \\
\hline
\end{tabular}

*Parameters were calculated using the data presented in Fig. 4 by a non-linear curve fitting algorithm (Prism 4.0).

A careful analysis of Fig. 4 and Table 3 reveals that the major discrepancy between the $\mathrm{SS}_{1 / 2}$ parameters calculated by the two approaches is mainly related to the basic assumptions of the equations. The SB equation treats the EI-SS data as having a sigmoidal shape [11], yet for hypotonic and hypertonic conditions the curves are not sigmoidal and only exhibit a plateau at higher shear stresses. However, the SB approach forces the data to be sigmoidal at lower SS, thereby yielding highly negative EI values (i.e., negative $\mathrm{EI}_{\min }$ ). As a consequence, $\mathrm{SS}_{1 / 2}$ for the $411 \mathrm{mOsm} / \mathrm{kg}$ data is essentially zero and is at a very low value for $164 \mathrm{mOsm} / \mathrm{kg}$; such remarkably low $\mathrm{SS}_{1 / 2}$ values occur since, by definition, they are the SS where $\mathrm{EI}$ is halfway between $\mathrm{EI}_{\max }$ and $\mathrm{EI}_{\min }$. Obviously, negative EI values are meaningless in the practice of normal ektacytometry. These results reinforce the observation that the SB method may not be useful if SS-EI curves significantly deviate from a sigmoid shape; such deviations might be quite common for abnormal RBC.

An alternative approach to using the $\mathrm{SB}$ equation is to do the calculations without the $\mathrm{EI}_{\min }$ parameter (i.e., assuming that $\mathrm{EI}_{\min }$ is zero); this approach yields more reasonable $\mathrm{EI}_{\max }$ and $\mathrm{SS}_{1 / 2}$ parameters (compare Tables 3 and 4). It is likely that the negative EI values reflect a problem of RBC orientation at low SS, including initial non-alignment with the shear field and/or a lack of time for the cell to reach a stable equilibrium shape after being deformed at a higher SS; obviously, negative EI values do not mean an actual change in elongation [11]. Thus, the three-parameter SB model might be useful in the usual protocol for RBC laser-diffraction ektacytometry: the measurements start with very low SS at which EI is close to zero, and SS is increased gradually.

\section{Problems in detecting alterations in deformability using various approaches}

It is obvious from the above examples that a complete approach to SS-EI curves requires the evaluation of all parameters contributing to the model equation used in curve fitting: the two parameters for LB and $\mathrm{EH}$ approaches and the four for the SB approach. Even a parameter determined by the LB equation cannot be directly compared between two groups of data if the other parameter is also changed. This situation is best illustrated by the influence of osmolality alterations (Fig. 4 and Table 3). The significantly reduced $\mathrm{SS}_{1 / 2}$ for cells in hypertonic media does not mean that $\mathrm{RBC}$ deformability is improved since it is important to also consider the extreme reduction in $\mathrm{EI}_{\max }$. This problem is even more exaggerated if the SB approach was used, due to the influence of the other parameters (especially $\mathrm{EI}_{\mathrm{min}}$, see Table 3). Thus, given such extreme alterations of SS-EI curves, data reduction approaches might be misleading if the parameters are evaluated in isolation. 
Table 4

Parameters for effects of osmolality obtained with the $\mathrm{SB}$ approach assuming $\mathrm{EI}_{\min }$ is zero*

\begin{tabular}{lccc}
\hline & \multicolumn{3}{c}{ Streekstra-Bronkhorst } \\
\cline { 2 - 4 } & $295 \mathrm{mOsm} / \mathrm{kg}$ & $164 \mathrm{mOsm} / \mathrm{kg}$ & $411 \mathrm{mOsm} / \mathrm{kg}$ \\
\hline $\mathrm{EI}_{\max }$ & 0.613 & 0.406 & 0.215 \\
$\mathrm{SS}_{1 / 2}$ & 2.883 & 0.845 & 1.773 \\
$\mathrm{~m}$ & 1.252 & 1.270 & 0.696 \\
\hline
\end{tabular}

*Parameters calculated for the data presented in Fig. 4 using the $\mathrm{SB}$ equation with $\mathrm{EI}_{\min }$ set to zero.

It can be argued, and is mathematically correct, that the relationship between the two parameters (e.g., SS and EI) can best be described with models taking into account all aspects of the relationship, and hence models with more parameters may be "better". However, some assumptions of the models may not always be strictly correct (e.g., the shape of the curve as discussed in Sec. 3), and the generated results might be seriously misleading. Further, having more than one parameter to compare, especially if the two parameters are interdependent, may not sufficiently simplify the evaluation process, but rather may even further complicate interpretation of the data. A reduced $\mathrm{SS}_{1 / 2}$ often indicates improved RBC deformability, yet a decreased $\mathrm{SS}_{1 / 2}$ might also be related to a reduced $\mathrm{EI}_{\max }$ and may therefore reflect greatly reduced RBC deformability rather than improvement. This difficulty in interpretation may only be obvious if two or more parameters are evaluated together; if not so evaluated, the major aim of the parameterization procedure (i.e., accurate numerical description of SS-EI curve with a single parameter) may not be realized.

\section{Solutions for the described problems}

It is clear from the above discussion and from previously published data $[2,11]$ that parameterization models should avoid using a graphical method based on the slope and intercept of a line for obtaining the parameters of interest. In particular, the LB approach may involve significant errors if $\mathrm{EI}_{\max }$ and $\mathrm{SS}_{1 / 2}$ are derived by linear regression and graphical extrapolation [2]. Such problems do not exist if the models are applied using non-linear curve fitting algorithms; these methods are currently available from several commercial sources (e.g., Prism 4.0, GraphPad Software Inc., La Jolla, CA). When using these algorithms, the three approaches described above do not mathematically differ, provided the limitations noted for the SB approach are considered.

Based on the analysis of results detailed in Tables 1, 2 and 3, it is strongly recommended that $\mathrm{EI}_{\text {min }}$ should not be considered and that only the three-parameter version of the $\mathrm{SB}$ model (i.e., $\mathrm{EI}_{\min }$ is zero) be used. However, under special circumstances (e.g., determining the SS-EI curve starting from high SS or the existence of negative EI values in the data set), the four-parameter version may be useful. Although the exponent " $m$ " of the SB model is usually close to unity for normal RBC, it can differ for SS-EI curves obtained with abnormal RBC: the SB model's " $m$ " might be especially useful for clinical studies [11]. Salgado et al. reported that the SB model used for calculating $\mathrm{EI}_{\max }$ and $\mathrm{SS}_{1 / 2}$ was superior in detecting differences of RBC deformability in septic patients [16]. A similar conclusion was reached in an experimental study where the SB model was slightly superior to the LB model in detecting differences between control and septic rats [3]. Alternatively, the LB model has been shown to have a slightly higher power for detecting alterations in RBC deformability caused by low-concentrations of glutaraldehyde [2]. 
Table 5

Comparison of $\mathrm{SS}_{1 / 2}, \mathrm{EI}_{\max }$ and $\mathrm{SS}_{1 / 2} / \mathrm{EI}_{\max }$ for $\mathrm{RBC}$ subjected to $100 \mathrm{~Pa}$ shear stress for 300 seconds*

\begin{tabular}{lcccc}
\hline & Control & Mechanical stress & $p$ & Standardized difference \\
\hline \multicolumn{5}{c}{ Lineweaver-Burke } \\
$\mathrm{SS}_{1 / 2}$ & $3.430 \pm 0.144$ & $3.769 \pm 0.109$ & $>0.05$ & 0.706 \\
$\mathrm{EI}_{\text {max }}$ & $0.651 \pm 0.002$ & $0.526 \pm 0.017$ & $<0.001$ & 2.808 \\
$\mathrm{SS}_{1 / 2} / \mathrm{EI}_{\text {max }}$ & $5.263 \pm 0.206$ & $7.303 \pm 0.361$ & $<0.001$ & 1.856 \\
\multicolumn{5}{c}{ Streekstra-Bronkhorst } \\
$\mathrm{SS}_{1 / 2}$ & $2.299 \pm 0.101$ & $6.836 \pm 0.653$ & $<0.001$ & 2.596 \\
$\mathrm{EI}_{\max }$ & $0.662 \pm 0.003$ & $0.633 \pm 0.022$ & $>0.05$ & 0.483 \\
$\mathrm{SS}_{1 / 2} / \mathrm{EI}_{\max }$ & $3.480 \pm 0.164$ & $10.828 \pm 1.001$ & $<0.001$ & 2.739 \\
\hline
\end{tabular}

*Calculations based on data presented in Fig. 3 and Table 2. Standardized difference is the measure of the power of each parameter in detecting a given difference between the groups (e.g., Control and Mechanical Stress).

\section{Consideration of $\mathrm{SS}_{1 / 2} / \mathrm{EI}_{\max }$ ratio}

Data reduction methods applied to SS-EI curves should aim at providing a single parameter for comparisons between groups (e.g., control and patient groups in clinical studies). Therefore, reduction of the entire set of SS-EI pairs into one representative parameter is desirable. It is thus interesting to consider expressing $\mathrm{SS}_{1 / 2}$ in a standardized or normalized form by dividing $\mathrm{SS}_{1 / 2}$ by $\mathrm{EI}_{\max }$ (i.e., $\mathrm{SS}_{1 / 2} / \mathrm{EI}_{\max }$ ), thereby yielding a parameter which helps compensate for alterations of $\mathrm{EI}_{\max }$. It should be noted that the $\mathrm{SS}_{1 / 2} / \mathrm{EI}_{\max }$ ratio is the slope of the line in the LB model when 1/EI is plotted against 1/SS (see Fig. 1a). The ratio thus reflects the dependence of $\mathrm{EI}$ on $\mathrm{SS}$ independent of $\mathrm{EI}_{\max }$ alterations. As discussed above (Table 3), the $\mathrm{SS}_{1 / 2}$ parameter calculated by $\mathrm{LB}$ model and the $\mathrm{EI}_{\max }$ parameter calculated by the $\mathrm{SB}$ model failed to detect the significant change of RBC deformability induced by mechanical stress. However, the $\mathrm{SS}_{1 / 2} / \mathrm{EI}_{\max }$ ratio was markedly changed from Control when calculated using either model and both approaches yielded reasonable standardized differences (Table 5). Standardized differences were calculated by dividing the difference between the means of the two groups (i.e., Control and Mechanical Stress) by the pooled standard deviation (i.e., the square root of the mean of the squared standard deviations of the two groups) [5]. Standardized difference reflects the power of an applied method to detect a difference between two groups [21].

\section{Conclusion}

The data reduction methods for ektacytometry described herein are mathematically similar, but may be prone to various errors if not applied with caution. It is strongly recommended that all models (i.e., LB, EH and SB) should be utilized using a non-linear curve fitting algorithm and not by graphical extrapolations. The ultimate aim of these models is to reduce an entire data set to one parameter that is comparable among groups of patients or experiments. However, being faced with values for $\mathrm{EI}_{\max }$ and $\mathrm{SS}_{1 / 2}$ for each group (e.g., control vs treatment) after data reduction does not aid such comparisons. This situation is especially problematic if the change in one parameter (e.g., $\mathrm{EI}_{\max }$ ) affects another one (e.g., $\mathrm{SS}_{1 / 2}$ ). It is suggested that this difficulty may be avoided by using the $\mathrm{SS}_{1 / 2} / \mathrm{EI}_{\max }$ ratio as a single 
parameter since it has similar power (i.e., standardized difference) as $\mathrm{SS}_{1 / 2}$ and $\mathrm{EI}_{\max }$ and is more robust in reflecting alterations of deformability.

\section{References}

[1] O.K. Baskurt, M. Boynard, G.R. Cokelet, P. Connes, B.M. Cooke, S. Forconi, F. Liao, M.R. Hardeman, F. Jung, H.J. Meiselman, G.B. Nash, N. Nemeth, B. Neu, B. Sandhagen, S. Shin, G.B. Thurston and J.L. Wautier, New guidelines for hemorheological laboratory techniques, Clin Hemorheol Microcirc 42 (2009), 75-97.

[2] O.K. Baskurt, M.R. Hardeman, M. Uyuklu, P. Ulker, M. Cengiz, N. Nemeth, S. Shin, T. Alexy and H.J. Meiselman, Parametrization of red blood cell elongation index-shar stress curves obtained by ektacytometry, Scand J Clin Lab Invest 69 (2009), 777-788.

[3] O.K. Baskurt and H.J. Meiselman, Analyzing shear stress-elongation index curves: Comparison of two approaches to simplify data presentation, Clin Hemorheol Microcirc 31 (2004), 23-30.

[4] P.J.H. Bronkhorst, E.J. Nijhof and J.J. Sixma, Parametrization of the deformation curve as a tool for standardization and interpretation of ektacytometric measurements, Clin Hemorheol 15 (1995), 803-816.

[5] J. Cohen, Statistical power analysis for the behavioral sciences, Lawrance Earlbaum Associates, Hillsdale, NJ, 1988.

[6] M.R. Condon, J.E. Kim, E.A. Deitch, G.W. Machiedo and Z. Spolarics, Appearance of an erythrocyte population with decreased deformability and hemoglobin content following sepsis, Am J Physiol Heart Circ Physiol 284 (2003), H2177-H2184.

[7] Y. Du, W. Yao, Y. Qian, M. Han, Z. Wen and L. Ma, Hemorheological changes in patients with living-donor renal transplantation, Clin Hemorheol Microcirc 47 (2011), 199-209.

[8] M.R. Hardeman, P.T. Goedhart, J.G.G. Dobbe and K.P. Lettinga, Laser-assisted optical rotational cell analyzer (lorca). 1. A new instrument for measurement of various structural hemorheological parameters, Clin Hemorheol 14 (1994), 605-618.

[9] B.H.J. Hofstee, Non-inverted versus inverted plots in enzyme kinetics, Nature 184 (1959), $1296-1298$.

[10] R.M. Johnson, Ektacytometry of red blood cells, Methods in Enzymology 173 (1989), 35-54.

[11] P. Kenyeres, M. Rabai, A. Toth, G. Kesmarky, Z. Marton, T. Alexy and K. Toth, Reviewing data reduction methods for ektacytometry, Clin Hemorheol Microcirc 47 (2011), 143-150.

[12] R. Kotan, N. Nemeth, F. Kiss, J. Posan, K. Miszti-Blasius, L. Toth, I. Furka, I. Miko, P. Sapy and Z. Szentkereszty, Micro-rheological changes during experimental acute pancreatitis in the rat, Clin Hemorheol Microcirc 51 (2012), $255-264$.

[13] H. Lineweaver and D. Burk, The determination of enzyme dissociation constants, J Am Chem Soc 56 (1934), 658-666.

[14] N. Nemeth, F. Kiss, T. Hever, E. Brath, E. Sajtos, I. Furka and I. Miko, Hemorheological consequences of hind limb ischemia-reperfusion differs in normal and gonadectomized male and female rats, Clin Hemorheol Microcirc 50 (2012), 197-211.

[15] M. Piagnerelli, H. Njimi, T.V. Coelho, G. Reggiori, D. Castanares Zapatero, K. Donadello and J.L. Vincent, Limited effects of activated protein C on red blood cell deformability, Clin Hemorheol Microcirc (2012), DOI: 10.3233/CH-20121550

[16] D. Salgado, R. Favory, G. Reggiori, J.L. Vincent and M. Piagnerelli, Comparison of different methods to assess red blood cell deformability in critically ill patients, Crit Care Med 37S (2009), A127.

[17] H. Schmid-Schoenbein, P. Ruef, O. Linderkamp and diffractometer The shear stress, Rheodyne SSD for determination of erythrocyte deformability. I. Principles of operation and reproducibility, Clin Hemorheol 16 (1996), 745-748.

[18] S. Shin, Y.H. Ku, M.S. Park and J.S. Suh, Slit-flow ektacytometer: Laser diffraction in a slit rheometer, Cytometry 65B (2005), 6-13

[19] H. Stadnick, R. Onell, J.P. Acker and J.L. Holovati, Eadie-Hofstee analysis of red blood cell deformability, Clin Hemorheol Microcirc 47 (2011), 229-239.

[20] G.J. Streekstra, J.J. Zwaginga, E.J. Nijhof, J.J. Sixma and R.M. Heethaar, The relation between blood viscosity and plasma viscosity in the nephrotic syndrome, Clin Hemorheol 14 (1994), 769-778.

[21] J. Stuart, P.C.W. Stone, G. Freyburger, M.R. Boisseau and D.G. Altman, Instrument precision and biological variability determine the number of patients required for rheological studies, Clin Hemorheol 9 (1989), 181-197.

[22] A. Vaya, J.M. Fuente, M. Montero, R. Perez and J.M. Ricart, Erythrocyte deformability in naive HIV-infected patients, Clin Hemorheol Microcirc 51 (2012), 235-241. 
Copyright of Clinical Hemorheology \& Microcirculation is the property of IOS Press and its content may not be copied or emailed to multiple sites or posted to a listserv without the copyright holder's express written permission. However, users may print, download, or email articles for individual use. 